\section{Garrod, Sir Archibald Edward}

H. Fiedler

Erfurt, Deutschland

Lebensdaten Britischer Mediziner und Biochemiker, geboren 1857 in London, gestorben 1936 in Cambridge. Neben seiner Praxis war er 20 Jahre an verschiedenen Londoner Kliniken und 1920-1927 als Regius Professor of Medicine an der Universität Oxford tätig. Garrod propagierte die wissenschaftliche Forschung als Basis einer medizinischen Tätigkeit. Er war befreundet mit Nobelpreisträger F.G. Hopkins (Stoffwechsel der Muskeln, Milchsäureproduktion, Glutathion, Vitamine). Nach Wiederentdeckung der Mendel-Regeln um 1900 und durch Zusammenarbeit mit dem Genetiker W. Bateson (prägte 1905 den Begriff Genetik) erkannte er die Ursachen genetischer Krankheiten.

Verdienste Durch Verknüpfung von chemischen Bestandteilen im Urin mit der biochemischen Pathologie und den
Verwandtschaftsverhältnissen der Patienten entdeckte er mehrere angeborene metabolische Krankheiten: $>$ Alkaptonurie ( $\triangleright$ Alkapton), Hämatoporphyrie, kongenitale Steatorrhoe, Cystinurie, Pentosurie und Albinismus. 1908 fasste er diese Krankheiten als „Inborn Errors of Metabolism“ zusammen und betonte die biochemische Individualität: „Jeder Mensch unterscheidet sich vom anderen durch den Enzym (Gen)-Besatz“ und kam zu der Hypothese „Ein Gen - Ein Enzym“. Erst nach 1910 wurde erkannt, dass Garrod ein wichtiges neues Gebiet der Medizin erschlossen hatte. Er erhielt viele Auszeichnungen und wurde in das Medical Research Council aufgenommen.

\section{Literatur}

Bearn AG (1996) Inborn errors of metabolism: Garrod's Legacy. Mol Med 2(3):271-273

Rolleston JD (2004) Garrod, Sir Archibald Edward (1857-1936), Physician and biochemist. Oxford Dictionary of National Biography. Oxford University Press. Oxford/New York 